\title{
Prevention of violent revictimization in depressed patients with an add-on internet-based emotion regulation training (iERT): study protocol for a multicenter randomized controlled trial
}

\author{
Carolien Christ ${ }^{1,2,3^{*}}$ (D) Marleen M. de Waal ${ }^{2,4}$, Digna J. F. van Schaik ${ }^{1,3}$, Martijn J. Kikkert ${ }^{2}$, Matthijs Blankers ${ }^{2,4,5}$,
} Claudi L. H. Bockting ${ }^{6}$, Aartjan T. F. Beekman ${ }^{1,3}$ and Jack J. M. Dekker ${ }^{2,7}$

\begin{abstract}
Background: Psychiatric patients are at high risk of becoming victim of a violent crime compared to the general population. Although most research has focused on patients with severe mental illness, depressed patients have been demonstrated to be prone to victimization as well. Victimization is associated with more severe symptomatology, decreased quality of life, and high risk of revictimization. Hence, there is a strong need for interventions that focus on preventing violent revictimization. Since emotion dysregulation is associated with both victimization and depression, we developed an internet-based Emotion Regulation Training (iERT) to reduce revictimization in depressed patients. This study aims to evaluate the clinical and cost-effectiveness of iERT added to Treatment As Usual (TAU) in reducing incidents of violent revictimization among depressed patients with a recent history of victimization. Furthermore, this study aims to examine secondary clinical outcomes, and moderators and mediators that may be associated with treatment outcomes.

Methods: In a multicenter randomized controlled trial with parallel group design, patients with a major depressive disorder and a history of violent victimization over the past three years $(N=200)$ will be allocated to either TAU + iERT $(N=100)$ or TAU only $(N=100)$, based on computer-generated stratified block randomization. Assessments will take place at baseline, 8 weeks, 14 weeks, and 6 months after start of treatment, and 12, 24, and 36 months after baseline. The primary outcome measure is the total number of violent victimization incidents at 12 months after baseline, measured with the Safety Monitor: an adequate self-report questionnaire that assesses victimization over the preceding 12 months. Secondary outcome measures and mediators include emotion dysregulation and depressive symptomatology. An economic evaluation with the societal perspective will be performed alongside the trial.

Discussion: This study is the first to examine the effectiveness of an intervention aimed at reducing violent revictimization in depressed patients. If effective, iERT can be implemented in mental health care, and contribute to the well-being of depressed patients. Furthermore, the results will provide insight into underlying mechanisms of revictimization. (Continued on next page)
\end{abstract}

\footnotetext{
* Correspondence: c.christ@ggzingeest.nl

'Department of Psychiatry, VU University Medical Center/ GGZ inGeest, P.O.

Box 7057, 1007 MB Amsterdam, The Netherlands

${ }^{2}$ Department of Research, Arkin Mental Health Care, Klaprozenweg 111, 1033

NN Amsterdam, The Netherlands

Full list of author information is available at the end of the article
} 
(Continued from previous page)

Trial registration: The study is registered at the Netherlands Trial Register (NTR5822). Date of registration: 4 April 2016.

Keywords: Victimization, Depression, Emotion regulation, iERT-training, E-mental health, Internet-based intervention, Violence prevention, Multicenter randomized controlled trial

\section{Background}

Psychiatric patients are at high risk of becoming victim of a violent crime [1, 2]. Prevalence rates of violent victimization - commonly defined as physical assault, sexual assault or threat of violence - in psychiatric patients worldwide have been demonstrated to be up to 11 times higher in comparison with the general population, with most studies reporting 3 to 6 -fold elevated odds [1-3]. Recently, a Dutch prevalence study conducted by Kamperman et al. (2014) [3] showed that $19.1 \%$ of outpatients with severe mental illness (SMI) had become victim of a violent crime over the past year, as compared to $6.1 \%$ of the general population. Whilst research has focused mainly on psychiatric patients as perpetrators (e.g., [4]), several studies have demonstrated that patients are more likely to be victim rather than perpetrator of a crime $[5,6]$.

Victimization is a highly stressful event that can aggravate existing symptoms [7] and substantially decrease quality of life [8] in psychiatric patients. Victimization is associated with physical injury, hospitalization $[7,8]$, treatment resistance [9], depression, posttraumatic stress disorder, and substance abuse [7]. Furthermore, initial victimization heightens the risk of future revictimization $[10,11]$, and therefore might induce a vicious cycle of stressful experiences and worsening symptoms. Due to increased service use, health care use, and productivity losses, victimization has a substantial economic impact as well [12].

To date, most studies have addressed victimization in patients with severe mental illness (SMI) $[6,13,14]$ and patients with substance use disorders $[15,16]$. Studies that specifically address victimization in other psychiatric populations, such as outpatients with affective disorders, are scarce. The only clinical study that focused on patients with depression revealed that depressed outpatients were 3.4 times more likely to be victim of a violent crime than members of the general population [17]. In a general population study of women, a current depression predicted subsequent physical victimization, but not subsequent sexual victimization [18]. Furthermore, a meta-analysis demonstrated depressed subjects to be vulnerable to domestic violence [19].

Despite the elevated prevalence rates and harmful consequences of victimization in psychiatric patients, evidence-based interventions specifically aimed at reducing victimization risk are not available. Currently, two interventions that aim to prevent victimization in patients with dual diagnosis [20] and psychotic disorders [21] are being examined in randomized controlled trials. There remains a strong need for evidence-based interventions that focus on reducing violent victimization in other high-risk psychiatric populations $[3,6,13,22]$, including depressed patients.

To be able to develop an intervention that specifically reduces victimization in depressed patients, knowledge regarding risk factors for victimization is necessary. Unfortunately, there remains a paucity of controlled, prospective studies that systematically examine risk factors, and no risk factors of victimization in depressed patients have yet been identified. Nevertheless, several mainly cross-sectional - studies have identified various factors that have consistently been associated with victimization and revictimization in patients with SMI: symptom severity $[2,23]$, alcohol and drug abuse $[2,23$, 24], a history of child abuse [23], and previous victimization [11]. In the general population, a history of child abuse [25], previous victimization [10], alcohol and drug abuse [26], and dysfunctional emotion regulation [27] have been associated with victimization risk.

Dysfunctional emotion regulation is considered to be both a consequence of prior victimization and a predictor of future revictimization [27, 28]. Emotion regulation refers to "the processes responsible for monitoring, evaluating, and modifying emotional reactions, especially their intensive and temporal features, to accomplish one's goals" [29]. Using a prospective design, Messman-Moore, Ward and Zerubavel (2013) [27] demonstrated emotion dysregulation to significantly predict subsequent revictimization in previously victimized female students. Several authors hypothesize that dysfunctional emotion regulation interferes with the ability to appraise risk situations [30-32], and may therefore impede self-protection and escape responses. Comparably, Marx et al. (2005) [28] suggested that victims of child sexual abuse adapt certain emotion regulation strategies - especially passive, avoidant behaviors - to cope with their increased level of fear and arousal. However, instead of reducing their psychological distress, the authors hypothesized that these strategies (1) reduce the effort that can be given to self-protection in dangerous situations, (2) impair risk assessment and threat detection, and (3) signal vulnerability to possible perpetrators, thereby increasing proneness to 
future victimization [28]. Hence, dysfunctional emotion regulation may be an important target for preventing revictimization.

Over the past decades, emotion dysregulation has been pointed out repeatedly as a perpetuating factor of depression. Difficulties in regulating negative emotions are associated with depressive symptoms in both cross-sectional and longitudinal studies [33-36]. Correspondingly, evidence of experimental studies suggests that a current or past depression is likely to coincide with emotion dysregulation [3740]. Changes in emotion regulation have been demonstrated to partially mediate treatment outcome in treatment for several disorders [41-43], including depression: Radkovsky et al. (2014) [35] demonstrated that the successful application of functional emotion regulation skills was associated with a reduction of depressive symptoms.

In conclusion, dysfunctional emotion regulation seems to be a promising target of intervention for both victimization and depression. Therefore, we developed an internet-based emotion regulation training (iERT) that will be added to Treatment As Usual (TAU). iERT is based on the Affect Regulation Training (ART) [44]: an intensive, structured group skills training that was demonstrated to be an effective addition to Cognitive Behavioral Therapy in decreasing depressive symptoms and enhancing various emotion regulation skills in depressed patients [45]. To provide an accessible and more feasible training that can be added to TAU, we developed a guided online version of ART that consists of 6 sessions. Numerous studies have demonstrated internetbased interventions to be an effective treatment for depression [46, 47]; moreover, interventions with online guided support have been found to be equally effective as face-to-face treatment $[48,49]$.

iERT is a transdiagnostic online add-on training that aims to enhance emotion regulation in patients by teaching them four emotion regulation skills: (1) non-judgmental awareness of emotions, (2) acceptance and tolerance of emotions, (3) analyzing emotions, and (4) modifying emotions. By enhancing emotion regulation skills, iERT aims to reduce violent victimization. Since both depressed patients and previously victimized patients are more likely to experience dysfunctional emotion regulation and have an increased revictimization risk compared to others, we will investigate the effectiveness of iERT in a high-risk population of previously victimized, depressed patients, who are specifically likely to benefit.

\section{Research aims}

The main purpose of this study is to evaluate the clinical effectiveness of the addition of iERT to Treatment As Usual (TAU) in reducing incidents of revictimization among patients with a major depressive disorder and a recent history of victimization. Since previous studies underline the hypothesis that ER represents an underlying mechanism leading to revictimization, we expect TAU + iERT to significantly decrease incidents of revictimization as compared to TAU alone, by enhancing ER skills.

In addition, we aim to examine the cost-effectiveness of the addition of iERT to TAU in reducing revictimization, and its effectiveness in reducing depressive symptoms and other secondary clinical outcomes. We expect the addition of iERT to significantly reduce depressive symptoms and to improve other secondary outcomes. Finally, we aim to enhance knowledge regarding the underlying mechanisms of victimization. Using a randomized, prospective, and longitudinal design, we will therefore examine the relation between victimization and changes in a variety of relevant secondary outcomes and potential mediators and moderators.

\section{Methods}

\section{Design}

We will conduct a multicenter two-arm randomized controlled trial (RCT) with a parallel group design, in which 200 participants will be allocated to either TAU $(N=100)$ or TAU $+\mathrm{iERT}(N=100)$ after the first baseline assessment. The effectiveness of the addition of iERT to TAU will be examined at 12 months after baseline assessment, and at follow-up 24 and 36 months after baseline assessment. Additional clinical outcome assessments will take place 8 weeks, 14 weeks, and 6 months after start of treatment. Apart from a diagnostic interview (Mini International Neuropsychiatric Interview; MINI) [50] that will be conducted at baseline and at 12 months follow-up, all assessments consist of self-report measures that will be completed over the Internet. The Medical Ethical Committee of the VU University Medical Center has approved the study protocol. The study is registered at the Netherlands Trial Register, part of the Dutch Cochrane Center (NTR5822).

\section{Participants}

Our target population consists of 200 adult outpatients with both a depression and a recent history of victimization. Inclusion criteria are: (1) a diagnosis of a major depressive disorder according to DSM-IV criteria, with or without a concurrent anxiety disorder other than Obsessive-Compulsive Disorder; (2) an indication for evidence-based psychotherapy for MDD or anxiety disorder according to clinical practice guidelines; (3) having been victim of at least one violent crime (physical assault, sexual assault, or threat) over the past three years; (4) access to a computer or tablet with Internet connection; and (5) an age of 18 years or older. Exclusion criteria are: (1) insufficient understanding of the spoken and written Dutch language; (2) psychotic symptoms; (3) bipolar disorder; (4) concurrent substance dependency 
that requires intervention; and (5) current high risk for suicide that requires intervention.

\section{Sample size}

Our primary outcome variable is the number of incidents of victimization (count data); therefore, a Poisson distribution is assumed. A priori sample size calculation was performed under this assumption, using the asypow package for $R$ 3.0. Since we are the first to examine effects of an intervention aimed at reducing revictimization in depressed patients, it is difficult to determine an exact estimate of the effect size for the main outcome measure. We expect that patients in the experimental condition will have experienced $30 \%$ less incidents at the endpoint in comparison with patients in the control condition, which we consider a realistic and clinically meaningful effect.

A previous study showed that $34 \%$ of depressed patients had been victim of at least one violent crime during one year [17]. On average, these patients had been victim of $3.30(\mathrm{SD}=4.32)$ violent crimes in a one-year period - after two outliers (2\%) who reported an extremely high number of incidents were deleted. Based on these results, we calculated that the expected average number of incidents over a period of 12 months after baseline, in a subsample of recently victimized patients, will be 3.0. Since the observed variance in the previous study indicated an overdispersed Poisson distribution, we performed numerical simulation in $\mathrm{R}$ for our sample size calculation, with adjustment for the expected overdispersion. Based on this simulation, we will need a minimum of 95 patients in each condition to demonstrate a difference between conditions of $30 \%$ in total incidents with $\alpha=.05$, two-sided, and $1-\beta=.80$. To account for missing information due to patient dropout, we aim to include 200 patients in total.

\section{Procedure \\ Recruitment and consent}

Participants will be recruited at the mood and anxiety disorder departments of GGZ inGeest and Arkin: the two largest mental health institutes in Amsterdam, the Netherlands. All patients referred to one of the participating sites will be screened for eligibility by a clinician during regular intakes, and all eligible patients will receive written study information directly after intake. Patients who agree to be approached by a researcher will be contacted by telephone after one week by a research assistant, who will provide further information about participation in the study and will make an appointment for the screening interview. The screening will preferably take place within one to maximum four weeks before the patient starts with TAU, and will be performed either by telephone or face-to-face - depending on the patient's preference.
At the screening, inclusion and exclusion criteria will be assessed in detail by a research assistant. First, the MINI International Neuropsychiatric Interview (MINI 5.0) [50] will be administered to assess a current diagnosis of depression and other Axis I disorders. In addition, three slightly adapted questions of the Safety Monitor addressing the experience of three types of violent crime (physical assault, sexual assault, or threat) will be asked. A definition and examples of each type of crime will be provided. If the patient reports having experienced at least one violent crime over the past three years, he or she will be asked to briefly describe the crime to ensure that he or she was violently victimized. All patients who meet the criteria will sign an informed consent prior to the first assessment, and will be included in the study. To decrease the burden for participants, the baseline assessment will be divided into two parts that both consist of online administered selfreport questionnaires. The first part is to be completed within one week after the screening, and the second part is to be completed preferably within a week after the first part. Figure 1 provides an overview of the trial design.

\section{Randomization and procedure}

Randomization will be carried out after completion of the first part of the baseline assessment. Randomization of participants in every participating site will be performed by a research associate of the data management department of GGZ inGeest, who is not familiar with the subject, nor involved in providing any kind of mental health care. Randomization will take place at an individual level, stratified by mental health care site, using a computer-generated block randomization schedule. To ensure that an equal number of patients will be allocated to TAU and TAU + iERT, the allocation ratio will be 1:1. To prevent selection bias, researchers and outcome assessors will be blind to block size and order, and will not have access to the randomization schedule. Due to the nature of treatments, blinding of participants and therapists to treatment condition is not feasible. Blinding research assistants to treatment allocation is not applicable to most outcome measures, since these will be administered online. Research assistants responsible for administering the MINI interview by telephone at 12 months after baseline will be blinded to treatment allocation. To promote data quality, all research assistants will be trained in administering the MINI interview. Audio recordings will be made of every MINI interview, which will be used in supervision sessions. Since all other measures concern web-based questionnaires that are filled out by the participants themselves, double data entry procedures are not applicable.

During the treatment phase, assessments will be administered at 8 weeks, 14 weeks, and 6 months after start of TAU in both the experimental and the control 


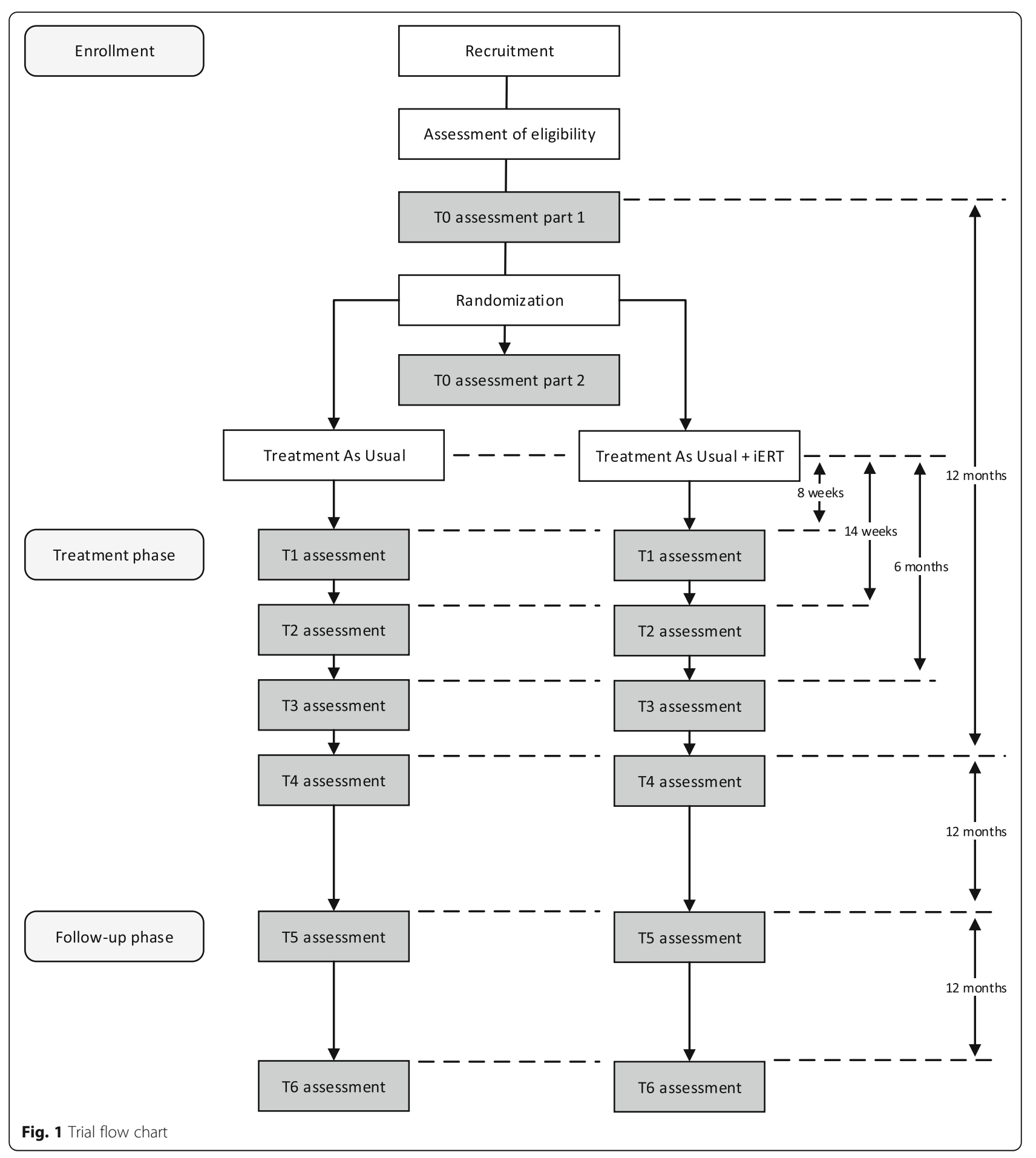

group. Follow-up assessments will be administered at 12, 24, and 36 months after the first baseline assessment. Except for the MINI, which will be assessed in a telephone interview at 12 months after baseline, all assessments will be conducted online. Patients receive an email with a link to the self-report questionnaires, and those who have not completed an assessment within
4 days will receive a reminder via e-mail. Patients who have not completed the assessments after one week will be approached via telephone by a research assistant. If necessary, online assessments can also be completed over the telephone by an assistant who is blinded to treatment condition. Participants will be compensated with a voucher of 15 euros per assessment for both the 
first and second part of T0, T1, T2, T3, T5 and T6, and a voucher of 30 euros for T4.

Additional questionnaires will be sent out in the TAU + iERT condition: a visual analogue mood scale (VAMS) is to be completed online after every iERT-session in the online treatment platform, and the System Usability Scale (SUS) and Technical Alliance Inventory (TAI) are to be filled out after completion of iERT. The SUS will also be sent out once to each iERT-therapist. Table 1 provides an overview of all instruments per assessment.

\section{Interventions}

\section{Internet-based emotion regulation training (iERT)}

The experimental intervention, iERT, is an abbreviated and slightly adapted online version of the Affect Regulation Training (ART) [44]. ART is a transdiagnostic structured group intervention that aims to enhance emotion regulation skills and consists of techniques from dialectical behavioral therapy, Systems Training for Emotion Predictability and Problem Solving program (STEPPS), emotion-focused therapy, Cognitive Behavioral Therapy (CBT), mindfulness-based interventions, self-compassion trainings, and problem-solving therapies. Although ART uses some techniques that are also included in CBT, ART explicitly and exclusively focuses on enhancing emotion regulation skills as opposed to CBT, which mainly targets cognitive and behavioral antecedents of depression [51]. In ART, the patient acquires seven emotion regulation skills, which the patient learns to use one-by-one by means of psycho-education and exercises. Subsequently, the patient practices each newly acquired skill in combination with previous skills using an audio file that guides him or her through the cycle of acquired skills [51].

To develop an online version of ART for patients with a major depressive disorder in specialized mental health care institutes in the Netherlands, ART was first translated into the Dutch language by an educated medical translator. Subsequently, the material was abbreviated and simplified, since all depression experts whom we consulted considered the original version too

Table 1 Overview of instruments per assessment

\begin{tabular}{|c|c|c|c|c|c|c|c|c|}
\hline Instrument & $\mathrm{TO}_{1}$ & $\mathrm{TO}_{2}$ & $\mathrm{~T} 1$ & T2 & T3 & T4 & T5 & $\overline{T 6^{\mathrm{a}}}$ \\
\hline Mini International Neuropsychiatric Interview & $x$ & & & & & $x$ & & \\
\hline Safety Monitor & $x$ & & & & & $x$ & $x$ & $x$ \\
\hline Inventory of Depressive Symptomatology & $x$ & & $x$ & $x$ & $x$ & $x$ & $x$ & $x$ \\
\hline Difficulties in Emotion Regulation Scale & $x$ & & $x$ & $x$ & $x$ & $x$ & $x$ & $x$ \\
\hline Visual Analogue Mood Scale & $x$ & & $x^{b}$ & $x$ & $x$ & $x$ & $x$ & $x$ \\
\hline Positive and Negative Affect Schedule & $x$ & & $x$ & $x$ & $x$ & $x$ & & \\
\hline Demographic questionnaire & $x$ & & & & & & & \\
\hline Posttraumatic Diagnostic Scale & & $x$ & & & $x$ & & & \\
\hline Childhood Trauma Questionnaire & $x$ & & & & & & & \\
\hline List of Threatening Experiences & & $x$ & & & & & & \\
\hline Brief Symptom Inventory & & $x$ & & & $x$ & & & \\
\hline Utrechts Coping List & & $x$ & & & & $x$ & & \\
\hline Ruminative Response Scale & & $x$ & $x$ & $x$ & $x$ & $x$ & & \\
\hline Pearlin Mastery Scale & & $x$ & $x$ & $x$ & $x$ & $x$ & & \\
\hline Dysfunctional Attitude Scale & & $x$ & $x$ & $x$ & $x$ & $x$ & & \\
\hline Self-Esteem Rating Scale & $x$ & & & & & & & \\
\hline Inventory of Interpersonal Problems & & $x$ & & & & $x$ & & \\
\hline NEO Five Factor Inventory & & & $x$ & & & & & \\
\hline EuroQol 5D-5 L & $x$ & & & & $x$ & $x$ & & \\
\hline Trimbos questionnaire on Costs associated with Psychiatric Illness & $x$ & & & & $x$ & $x$ & & \\
\hline Working Alliance Inventory & & & & $x$ & & & & \\
\hline Client Satisfaction Questionnaire & & & & $x$ & & & & \\
\hline Technical Alliance Inventory & & & & $x^{c}$ & & & & \\
\hline System Usability Scale & & & & $x^{c}$ & & & & \\
\hline
\end{tabular}


complicated and extensive for online use in moderately to severely depressed patients. iERT therefore focuses on a selection of four instead of seven skills: (1) non-judgmental awareness of emotions (Awareness); (2) acceptance and tolerance of emotions (Acceptance); (3) analysis of emotions (Analysis); and (4) active modification of emotions (Modulation). This selection was based on a literature review that we conducted, which indicated these emotion regulation skills to be highly relevant for both depressed patients $[33,35]$ and victimized patients [32, 52]. Lastly, in consultation with the developers of ART, we translated the Dutch, abbreviated protocol into an online version that will be provided on a secured online platform.

iERT consists of 6 sessions of approximately $30 \mathrm{~min}$. After each session, a trained psychologist will provide feedback and guidance using secured e-mail within the online platform. In iERT, general psycho-education about the four skills and the origin, functions, and characteristics of emotions is offered in videos. These videos take 3 to $8 \mathrm{~min}$ to watch, and are designed to provide information in a clear, comprehensible, and visually attractive manner. Each session starts with one or two short psycho-education videos, followed by an exercise. Each session ends with an audio file exercise that combines the newly acquired skill with the previous skills. The participant is stimulated to practice with the audio file exercise at least once per week. When participants have mastered the skills by means of insession theory, examples and exercises, their iERTtherapist stimulates them to start practicing their skills in daily life. The first iERT-session will be introduced in the experimental condition after the fourth session of TAU; from that moment on, the iERTsessions will run parallel to TAU. A new session will become available weekly, provided that the participant has completed the previous session. The therapists will monitor whether their patients have worked through the previous session in time, and will contact them within two weeks if they have not.

\section{Treatment as usual (TAU)}

TAU is defined as the routine care that participants receive when treated for a depression or anxiety disorder in outpatient mental healthcare. The type of treatment may vary, and may include all evidence-based types of psychotherapy that are part of the treatment guidelines for depression and anxiety disorders - combined with pharmacotherapy, if necessary. TAU will mainly consist of Cognitive Behavioral Therapy (CBT) [53] or Interpersonal Psychotherapy (IPT) [54], often combined with antidepressant medication. TAU will be offered at each participating site, and will not be interfered with during the study in both the experimental group and the control group. TAU may be offered individually or in a group, and face-to-face, online, or blended. We will closely monitor the type and amount of treatment through patient records, the patient's self-reported health care utilization, and a therapist questionnaire.

\section{iERT-therapists}

iERT will be provided by therapists of the e-mental health clinic of GGZ inGeest, who have ample experience with delivering internet-based treatment for depression. All therapists will receive an extensive training on how to deliver iERT - including how to use the online intervention and how to provide written feedback within the theoretical framework of ART. In order to ensure treatment fidelity, a detailed treatment manual with standardized written feedback templates will be available to guide therapists through the iERT-treatment. In addition, iERT-therapists will regularly receive supervision. A random sample of each therapist's written feedback will be analyzed and discussed in supervision sessions. For all patients, the iERT-therapist will be a different therapist than their TAU-therapist. Prior to iERT, the TAU-therapist provides necessary information regarding the patient's diagnosis and treatment progress to the iERT-therapist - a procedure for which the patient has provided written consent.

\section{Primary outcome measure Violent victimization}

Violent victimization will be measured with section 4 of the Safety Monitor (Dutch version: Veiligheidsmonitor) [55], which is developed by the Dutch Ministry of Security and Justice. The Safety Monitor strongly resembles the International Crime Victimization Survey (ICVS) [56] and is used by Statistics Netherlands (CBS) to measure victimization annually on a large scale. The Safety Monitor is an adequate self-report instrument that assesses victimization of 11 different crimes, subdivided into three categories: violent crimes, property crimes, and vandalism. In this study, we will only assess the violent crimes category, which consists of three subcategories: physical assault, sexual assault, and threat. Physical assault will be defined as deliberately hurting a person physically, with or without the use of a weapon. Sexual assault will be defined as unwanted sexual touching. Lastly, threat will be defined as threatening to hurt a person physically or sexually, without using actual violence. For each of these crimes, participants are asked whether they have become victim of that crime in the past 5 years. If so, participants are asked whether they have experienced that crime in the past 12 months, and, when answering affirmative, how frequently they experienced that crime in the past 12 months. The primary outcome measure is the total 
number of violent victimization incidents at 12 months after baseline (T4).

\section{Key secondary outcome measures Diagnosis of depression}

The presence of a current and lifetime diagnosis of depression in the past and present will be assessed with section $\mathrm{A}$ and $\mathrm{B}$ of the MINI International Neuropsychiatric Interview (MINI; version 5.0) [50]. The MINI is a structured, clinician-administered diagnostic interview that is widely used to assess the presence of psychiatric disorders based on the Diagnostic and Statistical Manual of Mental Disorders (Fourth edition; DSM-IV) and the International Classification of Diseases (Tenth revision; ICD-10). The MINI demonstrated a good Kappa score of 0.84 in diagnosing MDD, with a sensitivity of 0.96 and a specificity of 0.88 in comparison with the patient-rated version of the Structured Clinical Interview for DSM-IV (SCID) [57]. The validated Dutch version of the MINI [58] will be used.

At T0, sections A and B of the MINI will be conducted to assess current and lifetime depression. In addition, sections $\mathrm{C}, \mathrm{D}, \mathrm{I}, \mathrm{J}, \mathrm{K}$, and $\mathrm{L}$ of the MINI will be administered to assess the presence of suicidality, bipolar disorders, posttraumatic stress disorder, alcohol abuse or dependency, drug abuse or dependency, and psychotic disorders, respectively. At $\mathrm{T} 4$, section $\mathrm{A}$ will be administered again in a telephone interview, to determine remission rates of depression. In addition, the sections covering suicidality, posttraumatic stress disorder, and alcohol and drug abuse or dependency will be administered again.

\section{Depressive symptom severity}

Depressive symptoms will be assessed with the 30-item Inventory of Depressive Symptomatology - Self Report (IDS-SR; Dutch translation) [59, 60], which is a self-report questionnaire designed to measure depressive symptom severity. The IDS-SR includes all diagnostic DSM-IV criteria for major depressive disorder (MDD), as well as melancholic, atypical, and commonly associated symptoms for MDD (e.g., anxious mood, irritable mood). The IDS-SR covers five core symptom domains: vegetative symptoms, cognitive changes, mood disturbance, endogenous symptoms, and anxiety symptoms. All items are rated on a 4-point Likert scale from 0 to 3, and are equally weighted in the total score. The IDS-SR has highly acceptable psychometric properties and has been demonstrated to be sensitive to treatment effects in depressed outpatients [60-62]. The IDS-SR will also be used to determine response and remission rates. Response rates will be defined as a reduction in baseline total score of the IDS-SR of at least $50 \%$. Remission rates will be defined as the absence of a diagnosis of depression according to the MINI and an IDS-SR score of $<18$ [60].

\section{Other secondary outcome measures}

Other secondary outcome measures are:

- Quality of life, as measured with the EuroQol 5D (EQ-5D-5 L) [63];

- Healthcare costs and productivity losses/gains, as measured with the Trimbos/iMTA questionnaire

- on Costs associated with Psychiatric illness (TiC-P) [64];

- Psychopathology, as measured with the Brief Symptom Inventory (BSI) [65];

- PTSD symptomatology, as measured with the Posttraumatic Diagnostic Scale (PDS) [66];

- Interpersonal functioning, as measured with the Inventory of Interpersonal Problems (IIP-C) [67];

- Coping style, as measured with the subscales Active tackling and Avoidance of the Utrecht Coping List (UCL) [68].

\section{Process variables \\ Emotion dysregulation}

Emotion dysregulation will be measured with the Difficulties in Emotion Regulation Scale (DERS) [69]. The DERS is a 36-item self-report scale that assesses clinically relevant emotion regulation difficulties across various dimensions, represented in six subscales: non-acceptance of emotional responses, difficulty engaging in goal-directed behavior, impulse control difficulties, lack of emotional awareness, limited access to emotion regulation strategies, and lack of emotional clarity. The DERS has demonstrated high internal consistency, good test-retest reliability [69-71], and adequate construct and predictive validity [69].

\section{Positive and negative affect}

The 20-item Positive and Negative Affect Schedule (PANAS) [72] will be used to assess positive and negative affect at the time of assessment; both represented by 10 items that are rated on a 5-point Likert scale. Each item consists of an affective state, such as "excited", "proud", and "attentive" for the positive affect subscale, and "distressed", "ashamed", and "guilty" for the negative affect subscale. The PANAS has good internal consistency and validity [72]. The Dutch version [73] yields acceptable to good psychometric properties as well, with a Cronbach's alpha of .77 and .87 for the positive and negative affect subscales, respectively [74].

\section{Sad mood}

Sad mood will be measured by a digital version of the 1-item Visual Analogue Mood Scale (VAMS), which 
consists of a line that runs from zero to 100 , with the descriptors "happy" located on the left side and "sad" on the right side. A higher score represents a sadder mood. Patients are asked to rate their current mood by placing a cursor on the line, with the following instruction: "You can answer the following question by replacing a cursor on the line from 0 to 100 . At this moment, I feel...". The VAMS has been used previously in studies examining the influence of sad mood on relapse and recurrence of depression [75, 76]. In the experimental condition, the VAMS will be assessed after each iERT-session as well.

\section{Brooding}

The 5-item Brooding subscale of the Ruminative Response Scale (RRS; Dutch version) [77, 78] assesses the dysfunctional thinking pattern of drawing one's attention to problems and their consequences. For each item, respondents are asked to rate the extent to which it reflects their responses to sadness on a 5-point Likert scale. An exemplary item is "I think: why do I always react this way?". The Brooding subscale has satisfactory internal consistency (Cronbach's alpha = .77) [77] and predicted depression both cross-sectionally [79] and prospectively [77].

\section{Dysfunctional attitudes}

The Dysfunctional Attitude Scale form A (DAS-A) is a self-report scale designed to measure patterns of negative thinking in depressed patients [80], with good internal consistency and validity [81-83]. In this study, the shorter 17-item version will be used (DAS-A-17). Respondents are asked to what extent they agree with each of 17 dysfunctional assumptions on a 7-point Likert scale. A confirmatory factor analysis demonstrated the Dutch version of the DAS-A-17 to yield good psychometric properties in terms of reliability and convergent construct validity [84].

\section{Sense of control}

Sense of control is measured with the 7-item Mastery scale [85]. Mastery concerns the extent to which one perceives oneself to be in control of events and factors that influence one's life [85]. Respondents are asked to rate the extent to which they agree with seven statements on a 5-point Likert scale, for example: "There is really no way that I can solve some of the problems that I have." The Mastery scale has adequate psychometric properties [85, 86].

\section{Potential moderators}

- Demographic characteristics, collected at baseline;

- Childhood trauma, as measured with the Childhood Trauma Questionnaire (CTQ) [87];
- Alcohol and drug abuse or dependency, as measured with the MINI 5.0 [50];

- Self-esteem, as measured with the Self-Esteem Rating Scale Short-Form (SERS-SF) [88];

- Personality dimensions, as measured with the Neuroticism-Extraversion-Openness Five Factor Inventory (NEO-FFI) [89];

- Negative life events, as measured with the List of Threatening Experiences [90];

- Working alliance, as measured with the Working Alliance Inventory - Short Form (WAI-SF) [91].

\section{Other variables of interest}

\section{Treatment evaluation}

The 8-item Client Satisfaction Questionnaire (CSQ-8) [92] will be administered to assess patient satisfaction with treatment in both the experimental group and the control group. The CSQ has high internal consistency $(\alpha=.93)$ [93].

In addition, the 10-item System Usability Scale (SUS) [94] will be administered to participants receiving TAU + iERT, after they have finished iERT-treatment regularly or prematurely. The SUS assesses participants' subjective perception of the usability of a technology system. The questionnaire will be administered once to each iERT-therapist as well. The SUS has been demonstrated to be reliable and robust [95].

\section{Contextual information of victimization}

The Safety Monitor assesses contextual information about the most recent incident of each violent crime experienced in the past 12 months: where the incident took place, whether the participant knew the perpetrator and what their relationship was, and whether the participant reported the crime to the police. To obtain more detailed information on the most recent victimization incident, we extended the Safety Monitor with supplemental questions, such as: "did you use substances or alcohol prior to the incident?".

\section{Victimization of non-violent crimes}

Apart from violent crimes, we will ask participants whether and how frequently they have experienced the following non-violent crimes in the past 12 months: burglary, car theft or theft of another motor vehicle, pickpocketing or non-violent robbery, theft of other property, fraud, and vandalism.

\section{Perpetration}

Perpetration will be measured with the extended version of the Safety Monitor. For each violent crime, participants will be asked whether they have ever committed that crime. If so, participants are asked whether and how frequently they have permitted that crime in the past 
12 months. For each crime they committed in the past 12 months, they will be asked whether they simultaneously were both victim and perpetrator, and if so, whether they committed the crime in self-defense. Prior to these questions, participants are reminded about the confidentiality of the assessment.

\section{Safety perception and perceived controllability of victimization}

Section 3 of the Safety Monitor assesses safety perception by asking participants whether and how often they feel unsafe. We extended this section with four questions regarding safety perception and perceived controllability of victimization, such as: "To what extent do you consider yourself able to prevent ending up in hazardous situations?" and "How likely do you think it is that you will fall victim to each of the following during the next twelve months?".

\section{Data analysis}

\section{Effectiveness}

Primary data analyses will be conducted in conformity with the intention-to-treat paradigm. In addition, perprotocol analyses will be performed. Missing data will be addressed using multiple imputation. Treatment effect regarding the primary outcome measure will be modeled with Generalized Linear Mixed Models (GLMM) with a Poisson distribution, since the primary outcome measure consists of count data. Treatment effect concerning the secondary outcome variables will be modeled using GLMM as well, taking into account distributional characteristics of the data. Considering the expected skewness of the data, we assume that removal of outliers will be necessary.

To assess the magnitude of treatment effects on primary and secondary outcome measures, Cohen's d between groups effect sizes for each time point will be calculated. Effect sizes of $\mathrm{d}=.9$ are considered large, effect sizes of $\mathrm{d}=.45$ are considered moderate and effect sizes of $d=.15$ are considered small [96]. Furthermore, multilevel mediation models will be used to examine various potential treatment mediators. Although the major question of this study concerns victimization, we will determine differences between both conditions regarding response and remission rates of depression as well.

\section{Cost-effectiveness}

The economic evaluation will be conducted alongside the randomized controlled trial and will be performed according to the intention-to-treat principle. With regard to the economic evaluation, we will take into account the CHEERS statement [97] and the 2015 ISPOR good research practices task force report on costeffectiveness analysis alongside clinical trials [98]. Using a societal perspective, we will evaluate the relationship between costs - direct medical costs, participant costs and productivity losses, as measured with the TiC-P - and health outcomes of TAU + iERT and TAU alone at 12 months after baseline.

We will take into account four types of costs: (1) the costs of offering the intervention (TAU + iERT or TAU only); (2) costs stemming from general health care uptake besides TAU + iERT or TAU only, including the costs of medication; (3) patients' out-of-pocket expenses (e.g. travel costs, leisure time spent on receiving care); and (4) costs stemming from productivity losses due to absenteeism or reduced efficiency while at work (presenteeism). Health care costs will be valued based on standard cost prices reported in the Dutch guideline for economic evaluation [99]. Costs due to productivity losses will be based on the gender- and age-specific labor costs. Data on resource use (health care uptake) and productivity losses will be collected with the widelyused TiC-P [64]. Using this approach, cumulative costs over the full trial period will be obtained from the cost estimates at times of the data collection waves.

We will perform both a cost-effectiveness analysis with incidents of victimization as effect measure and a costutility analysis using QALYs. QALYs will be based on preferences from general population samples to derive value sets to calculate the EQ-5D-5 L health utilities for the Netherlands. Using the area under the curve (AUC) method, the periods between the measurement waves will be multiplied by the utility of the health state in that period. This allows for the computation of quality adjusted life years (QALYs) over the entire trial period.

Incremental cost-effectiveness ratios (ICERs) and costeffectiveness acceptability curves (CEAC's) will be calculated. To estimate the uncertainty around the ICERs, 5000 bootstrapped samples will be extracted and plotted on cost-effectiveness planes. These data will also be used to plot Cost Effectiveness Acceptability Curves (CEACs). One-way sensitivity analyses and/or scenario analyses directed at assessing the impact of uncertainty in the main cost drivers will be performed to gauge the robustness of our findings. In addition, a sensitivity analysis in which covariate-adjusted CEACs can be constructed will be conducted using net benefit regression methods [100, 101].

\section{Data management}

Data management of this research project will be performed by the Data Management department of GGZ inGeest, which is not otherwise involved in the project. The project data will be securely saved on the central 
server of GGZ inGeest. The IT department professionally maintains the server and daily performs a backup of all data. All data will be pseudonymized using unique study codes that will be used to code and file all electronic information. Only designated members of the research team will have access to a secured file with the key that links this code to the participant's identity. Since all outcome measures concern either web-based questionnaires that are filled out by the participants themselves or a computer-based interview that is filled out digitally by a research assistant, hard copy storage of questionnaires is not applicable. All informed consents will be stored both electronically and in hard copy, with the hard copies stored in a locked cabinet at each participating site.

\section{Discussion}

This paper describes the study protocol of a randomized controlled trial aimed at assessing the effectiveness of the addition of iERT to TAU in reducing violent revictimization, depressive symptoms, and emotion regulation difficulties in previously victimized, depressed patients. Despite the high prevalence rates and societal burden of victimization in psychiatric patients, there remains a paucity of knowledge of risk factors and predictors regarding violent victimization in depressed patients. Accordingly, evidence-based interventions aimed at reducing victimization and revictimization are still scarce. To the best of our knowledge, this study is the first to examine the effectiveness of an intervention aimed at reducing revictimization in depressed patients. We will investigate the hypotheses that the addition of iERT to TAU is effective and cost-effective in decreasing incidents of revictimization in depressed patients compared to TAU alone. Furthermore, this study will examine whether the addition of iERT to TAU leads to a significant improvement on secondary outcome measures. If iERT is effective in reducing incidents of revictimization, it can be implemented in mental health care.

Major strengths of the current study are the long follow-up period of 36 months and the large amount of validated, clinically relevant outcome measures that are administered at multiple assessments during the treatment phase, which enables us to gain insight into the underlying mechanisms of revictimization. The most important concern of this study will be treatment adherence, since internet-based treatments are associated with high dropout levels [102, 103]. Weekly therapist guidance and the relatively small amount of iERT-sessions is expected to enhance treatment adherence, but it will remain an important challenge of this trial. To prevent study dropout, participants will receive monetary compensation for all assessments.
In conclusion, the prospective and longitudinal design of this study provides a unique opportunity to thoroughly examine revictimization rates and a variety of relevant secondary outcomes, mediators, and moderators over a period of three years.

\section{Abbreviations}

ART: Affect Regulation Training; AUC: Area Under the Curve; BSI: Brief Symptom Inventory; CBT: Cognitive Behavioral Therapy; CEAC: Cost-Effectiveness Acceptability Curves; CHEERS: Consolidated Health Economic Evaluation Reporting Standards; CSQ-8: 8-item Client Satisfaction Questionnaire; CTQ: Childhood Trauma Questionnaire; DAS-A: Dysfunctional Attitude Scale form A; DERS: Difficulties in Emotion Regulation Scale; EQ-5D-5 L: EuroQol 5D-5 L; ERT: Emotion Regulation Training; GLMM: General Linear Mixed Models; ICER: Incremental Cost-Effectiveness Ratio; ICVS: International Crime Victims Survey; IDS-SR: Inventory of Depressive Symptomatology - Self Report;

iERT: Internet-based Emotion Regulation Training; IIP-C: Inventory of Interpersonal Problems; ISPOR: International Society for Pharmaco-economics and Outcomes Research; MINI: Mini International Neuropsychiatric Interview; NEO-FFI: NEO Five Factor Inventory; PANAS: Positive and Negative Affect Schedule;

PDS: Posttraumatic Diagnostic Scale; PTSD: Post-Traumatic Stress Disorder QALY: Quality Adjusted Life Years; RCT: Randomized Controlled Trial;

RRS: Ruminative Response Scale; SERS-SF: Self-Esteem Rating Scale Short-Form; SMI: Severe Mental IIIness; STEPPS: Systems Training for Emotional Predictability and Problem Solving; SUS: System Usability Scale; TAl: Technical Alliance Inventory; TAU: Treatment As Usual; TiC-P : Trimbos/iMTA questionnaire on Costs associated with Psychiatric illness; UCL: Utrecht Coping List; VAMS: Visual Analogue Mood Scale; WAI-SF: Working Alliance Inventory - Short Form

\section{Acknowledgements}

This research is part of the Violence Against Psychiatric Patients programme, financed by the Netherlands Organisation for Scientific Research (NWO). We thank Adriaan Hoogendoorn (GGZ inGeest), who provided advice regarding the statistical analysis section.

\section{Funding}

The study is funded by the Netherlands Organisation for Scientific Research (NWO; grant number 432-13-811, awarded to AB, JD, MK, CB, DvS and CC). Contact information: Netherlands Organisation for Scientific Research,

Postbus 93510, 2509 AM Den Haag. The funding agency had no role in the planning of the study design and will not be involved in data collection and analysis, decision to publish or preparation of the manuscript.

\section{Availability of data and materials}

Researchers will only have restricted access to the data during the ongoing data collection. No interim analyses are planned, but cross-sectional comparisons of the baseline data will be conducted after inclusion of all participants. After completion of the study, only the Principal Investigator $(\mathrm{AB})$ and the Study Coordinator (CC) will have access to the data. Due to privacy concerns, raw data cannot be made openly accessible to the public, but data, statistical parameters and statistical code will be made accessible to interested researchers after request. We will promote dissemination of the results not only by means of scientific publications in international peer-reviewed journals and presentations at scientific conferences, but will also report our results to relevant stakeholders in newsletters and trial summaries. A written summary of the results will be made available to the participants after completion of the study. The publication of the results will be independent of the results, whether or not the experimental intervention is effective or cost-effective. Authorship eligibility will be decided according to the guidelines of the International Committee of Medical Journal Editors (ICMJE). All adverse events will be documented. In accordance with the national regulations in The Netherlands, data will be stored for 15 years.

\section{Authors' contributions}

CC drafted the manuscript, which was modified by MdW, MK, DvS, MB, CB, $A B$ and JD. CC and MB drafted the sections on costs, effects and cost-effectiveness calculations. All authors participated in the design of the study. All authors read and approved the final manuscript. 


\section{Ethics approval and consent to participate}

The study protocol has been reviewed and approved by the Medical Ethics Committee of the VU University Medical Center (registration number 2015.469). Participants will provide informed consent prior to enrollment in the study, and the informed consent form has been approved by the Medical Ethics Committee. The study is registered at the Netherlands Trial Register, part of the Dutch Cochrane Center (NTR5822). Approval for amendments to the protocol will be sought by the Ethics Committee, and will be updated in the Netherlands Trial Register. The study will be conducted in compliance with the Declaration of Helsinki and the Guidelines for Good Clinical Practice (GCP). The study protocol is in accordance with the Standard Protocol Items: Recommendations for Interventional Trials (SPIRIT) guidelines.

\section{Consent for publication}

Not applicable.

\section{Competing interests}

The authors declare that they have no competing interests.

\section{Publisher's Note}

Springer Nature remains neutral with regard to jurisdictional claims in published maps and institutional affiliations.

\section{Author details}

'Department of Psychiatry, VU University Medical Center/ GGZ inGeest, P.O. Box 7057, 1007 MB Amsterdam, The Netherlands. ${ }^{2}$ Department of Research, Arkin Mental Health Care, Klaprozenweg 111, 1033 NN Amsterdam, The Netherlands. ${ }^{3}$ Amsterdam Public Health research institute, VU University Medical Center, Van der Boechorststraat 7, 1081 BT Amsterdam, The Netherlands. ${ }^{4}$ Academic Medical Center, Department of Psychiatry, Amsterdam Institute for Addiction Research, University of Amsterdam, Meibergdreef 9, 1105 AZ Amsterdam, The Netherlands. ${ }^{5}$ Trimbos Institute Netherlands Institute of Mental Health and Addiction, Da Costakade 45, 3521 VS Utrecht, The Netherlands. ${ }^{6}$ Department of Clinical Psychology, Faculty of Social and Behavioural Sciences, University Utrecht, Heidelberglaan 1, 3584 CS Utrecht, The Netherlands. 'Department of Clinical Psychology, Neuro- and Developmental Psychology, Faculty of Behavioural and Movement Sciences, Vrije Universiteit Amsterdam, Van der Boechorststraat 1, BT 1081 Amsterdam, The Netherlands.

Received: 6 September 2017 Accepted: 17 January 2018

Published online: 02 February 2018

\section{References}

1. Teplin LA, McClelland GM, Abram KM, Weiner DA. Crime victimization in adults with severe mental illness: comparison with the National Crime Victimization Survey. Arch Gen Psychiatry. 2005;62:911-21.

2. Khalifeh H, Oram S, Osborn D, Howard LM, Johnson S. Recent physical and sexual violence against adults with severe mental illness: a systematic review and meta-analysis. Int Rev Psychiatry. 2016;28:433-51.

3. Kamperman AM, Henrichs J, Bogaerts S, Lesaffre EM, Wierdsma Al, Ghauharali RR, et al. Criminal victimisation in people with severe mental illness: a multi-site prevalence and incidence survey in the Netherlands. PLoS One. 2014;9:e91029.

4. Fazel S, Wolf A, Chang Z, Larsson H, Goodwin GM, Lichtenstein P. Depression and violence: a Swedish population study. Lancet Psychiatry. 2015;2:224-32

5. Choe JY, Teplin LA, Abram KM. Perpetration of violence, violent victimization, and severe mental illness: balancing public health concerns. Psychiatr Serv. 2008;59:153-64.

6. Maniglio R. Severe mental illness and criminal victimization: a systematic review. Acta Psychiatr Scand. 2009;119:180-91.

7. Resnick HS, Acierno R, Kilpatrick DG. Health impact of interpersonal violence. 2: medical and mental health outcomes. Behav Med. 1997;23:65-78.

8. Hanson RF, Sawyer GK, Begle AM, Hubel GS. The impact of crime victimization on quality of life. J Trauma Stress. 2010;23:189-97.

9. Neria Y, Bromet EJ, Carlson GA, Naz B. Assaultive trauma and illness course in psychotic bipolar disorder: findings from the Suffolk county mental health project. Acta Psychiatr Scand. 2005;111:380-3.
10. Roodman AA, Clum GA. Revictimization rates and method variance: a meta-analysis. Clin Psychol Rev. 2001;21:183-204.

11. Classen CC, Palesh OG, Aggarwal R. Sexual revictimization: a review of the empirical literature. Trauma Violence Abuse. 2005;6:103-29.

12. Robinson F, Keithley J. The impacts of crime on health and health services: a literature review. Health Risk Soc. 2000;2:253-66.

13. Latalova K, Kamaradova D, Prasko J. Violent victimization of adult patients with severe mental illness: a systematic review. Neuropsychiatr Dis Treat. 2014;10:1925-39.

14. de Mooij LD, Kikkert M, Lommerse NM, Peen J, Meijwaard SC, Theunissen J, et al. Victimisation in adults with severe mental illness: prevalence and risk factors. Br J Psychiatry. 2015;207:515-22.

15. Stevens A, Berto D, Frick U, Kerschl V, McSweeny T, Schaaf S, et al. The victimization of dependent drug users: findings from a European study, UK. Eur J Criminol. 2007:4:385-408.

16. Pirard S, Sharon E, Kang SK, Angarita GA, Gastfriend DR. Prevalence of physical and sexual abuse among substance abuse patients and impact on treatment outcomes. Drug Alcohol Depend. 2005;78:57-64.

17. Meijwaard SC, Kikkert M, de Mooij LD, Lommerse NM, Peen J, Schoevers RA, et al. Risk of criminal victimisation in outpatients with common mental health disorders. PLoS One. 2015:10:e0128508.

18. Acierno R, Resnick H, Kilpatrick DG, Saunders B, Best CL. Risk factors for rape, physical assault, and posttraumatic stress disorder in women: examination of differential multivariate relationships. J Anxiety Disord. 1999;13:541-63.

19. Trevillion K, Oram S, Feder G, Howard LM. Experiences of domestic violence and mental disorders: a systematic review and meta-analysis. PLoS One. 2012;7:e51740.

20. de Waal MM, Kikkert MJ, Blankers M, Dekker JJ, Goudriaan AE. Self-wise, otherwise, streetwise (SOS) training: a novel intervention to reduce victimization in dual diagnosis psychiatric patients with substance use disorders: protocol for a randomized controlled trial. BMC Psychiatry. 2015;15:267.

21. van der Stouwe EC, de Vries B, Aleman A, Arends J, Waarheid C, Meerdink $A$, et al. BEATVIC, a body-oriented resilience training with elements of kickboxing for individuals with a psychotic disorder: study protocol of a multi-center RCT. BMC Psychiatry. 2016;16:227.

22. Perese EF. Stigma, poverty, and victimization: roadblocks to recovery for individuals with severe mental illness. J Am Psychiatr Nursus Assoc. 2007;13: 285-95.

23. Goodman LA, Salyers MP, Mueser KT, Rosenberg SD, Swartz M, Essock SM, et al. Recent victimization in women and men with severe mental illness: prevalence and correlates. J Trauma Stress. 2001;14:615-32.

24. Hiday VA, Swartz MS, Swanson JW, Borum R, Wagner HR. Criminal victimization of persons with severe mental illness. Psychiatr Serv. 1999;50:62-8.

25. Messman-Moore TL, Long PJ. The role of childhood sexual abuse sequelae in the sexual revictimization of women: an empirical review and theoretical reformulation. Clin Psychol Rev. 2003;23:537-71.

26. Kilpatrick DG, Acierno R, Resnick HS, Saunders BE, Best CL. A 2-year longitudinal analysis of the relationships between violent assault and substance use in women. J Consult Clin Psychol. 1997;65:834-47.

27. Messman-Moore TL, Ward RM, Zerubavel N. The role of substance use and emotion dysregulation in predicting risk for incapacitated sexual revictimization in women: results of a prospective investigation. Psychol Addict Behav. 2013;27:125-32.

28. Marx BP, Heidt JM, Gold SD. Perceived uncontrollability and Unpredicability, self-regulation, and sexual Revictimization. Rev Gen Psychol. 2005;9:67-90.

29. Thompson RA. Emotion regulation: a theme in search of definition. Monogr Soc Res Child Dev. 1994;59(25-52):27-8.

30. Dietrich A. Childhood maltreatment and revictimization: the role of affect dysregulation, interpersonal relatedness difficulties and posttraumatic stress disorder. J Trauma Dissociation. 2007;8:25-51.

31. Messman-Moore TL, Walsh KL, DiLillo D. Emotion dysregulation and risky sexual behavior in revictimization. Child Abuse Negl. 2010;34:967-76.

32. Walsh $\mathrm{K}$, DiLillo D, Messman-Moore TL. Lifetime sexual victimization and poor risk perception: does emotion dysregulation account for the links? J Interpers Violence. 2012;27:3054-71.

33. Brockmeyer T, Bents $\mathrm{H}$, Holtforth MG, Pfeiffer N, Herzog W, Friederich HC. Specific emotion regulation impairments in major depression and anorexia nervosa. Psychiatry Res. 2012;200:550-3.

34. Berking M, Orth U, Wupperman P, Meier LL, Caspar F. Prospective effects of emotion-regulation skills on emotional adjustment. J Couns Psychol. 2008;55:485-94 
35. Radkovsky A, McArdle JJ, Bockting CL, Berking M. Successful emotion regulation skills application predicts subsequent reduction of symptom severity during treatment of major depressive disorder. J Consult Clin Psychol. 2014:82:248-62.

36. Berking M, Wirtz CM, Svaldi J, Hofmann SG. Emotion regulation predicts symptoms of depression over five years. Behav Res Ther. 2014:57:13-20.

37. Liverant Gl, Brown TA, Barlow DH, Roemer L. Emotion regulation in unipolar depression: the effects of acceptance and suppression of subjective emotional experience on the intensity and duration of sadness and negative affect. Behav Res Ther. 2008;46:1201-9.

38. Ehring T, Fischer S, Schnuelle J, Boesterling A, Tuschen-Caffier B. Characteristics of emotion regulation in recovered depressed versus never depressed individuals. Personal Individ Differ. 2008;44(7):1574-84.

39. Ehring T, Tuschen-Caffier B, Schnulle J, Fischer S, Gross JJ. Emotion regulation and vulnerability to depression: spontaneous versus instructed use of emotion suppression and reappraisal. Emotion. 2010;10:563-72.

40. Joormann J, Siemer M, Gotlib $\Vdash H$. Mood regulation in depression: differential effects of distraction and recall of happy memories on sad mood. J Abnorm Psychol. 2007;116:484-90.

41. Slee N, Spinhoven P, Garnefski N, Arensman E. Emotion regulation as mediator of treatment outcome in therapy for deliberate self-harm. Clin Psychol Psychother. 2008;15:205-16.

42. Wallace $L M$, Masson PC, Safer DL, von Ranson KM. Change in emotion regulation during the course of treatment predicts binge abstinence in guided self-help dialectical behavior therapy for binge eating disorder. J Eat Disord. 2014;2:35.

43. Gratz KL, Bardeen JR, Levy R, Dixon-Gordon KL, Tull MT. Mechanisms of change in an emotion regulation group therapy for deliberate self-harm among women with borderline personality disorder. Behav Res Ther. 2015; 65:29-35.

44. Berking M. Training emotionaler Kompetenzen. Heidelberg: Springer; 2008.

45. Berking $M$, Ebert $D$, Cuijpers $P$, Hofmann SG. Emotion regulation skills training enhances the efficacy of inpatient cognitive behavioral therapy for major depressive disorder: a randomized controlled trial. Psychother Psychosom. 2013;82:234-45.

46. Spek V, Cuijpers P, Nyklicek I, Riper H, Keyzer J, Pop V. Internet-based cognitive behaviour therapy for symptoms of depression and anxiety: a meta-analysis. Psychol Med. 2007;37:319-28.

47. Andersson G, Cuijpers P. Internet-based and other computerized psychological treatments for adult depression: a meta-analysis. Cogn Behav Ther. 2009;38:196-205

48. Cuijpers P, Donker T, van SA LJ, Andersson $G$. Is guided self-help as effective as face-to-face psychotherapy for depression and anxiety disorders? A systematic review and meta-analysis of comparative outcome studies. Psychol Med. 2010;40:1943-57.

49. Andersson G, Cuijpers P, Carlbring P, Riper H, Hedman E. Guided internetbased vs. face-to-face cognitive behavior therapy for psychiatric and somatic disorders: a systematic review and meta-analysis. World Psychiatry. 2014;13:288-95.

50. Sheehan DV, Lecrubier Y, Sheehan KH, Amorim P, Janavs J, Weiller E, et al. The Mini-international neuropsychiatric interview (M.I.N.I.): the development and validation of a structured diagnostic psychiatric interview for DSM-IV and ICD-10. J Clin Psychiatry. 1998;59(Suppl 20):22-33.

51. Berking M, Whitley B. Affect Regulation Training: A Practitioners' Manual. Springer; 2014.

52. Walsh K, DiLillo D, Scalora MJ. The cumulative impact of sexual revictimization on emotion regulation difficulties: an examination of female inmates. Violence Against Women. 2011;17:1103-18.

53. Beck AT, editor: Cognitive therapy of depression. Guilford press; 1979.

54. Klerman GL, Weissman MM: Interpersonal psychotherapy of depression: A brief, focused, specific strategy. Jason Aronson, Incorporated; 1994.

55. Akkermans M, Coumans M, Kloosterman R, Linden G, Moons E. Veiligheidsmonitor 2013. 3-3-2014. Den Haag, Centraal Bureau voor de Statistiek.

56. Killias M. International Crime Victimization Survey (ICVS). Encyclopedia of Victimology and Crime Prevention. Thousand Oaks: SAGE Publications, Inc; 486-489. 2010

57. Sheehan DV, Lecrubier $Y$, Sheehan $\mathrm{KH}$, Janavs J, Weiller E, Keskiner A, et al The validity of the MINI international neuropsychiatric interview (MINI) according to the SCID-P and its reliability. European Psychiatry. 1997;12: 232-41.
58. van Vliet IM, de Beurs E. The MINI-international neuropsychiatric interview. A brief structured diagnostic psychiatric interview for DSM-IV en ICD-10 psychiatric disorders. Tijdschr Psychiatr. 2007;49:393-7.

59. Rush AJ, Giles DE, Schlesser MA, Fulton CL, Weissenburger J, Burns C. The inventory for depressive Symptomatology (IDS): preliminary findings. Psychiatry Res. 1986;18:65-87.

60. Rush AJ, Gullion CM, Basco MR, Jarrett RB, Trivedi MH. The inventory of depressive Symptomatology (IDS): psychometric properties. Psychol Med. 1996;26:477-86

61. Rush AJ, Carmody T, Reimitz P. The inventory of depressive Symptomatology (IDS): clinician (IDS-C) and self-report (IDS-SR) ratings of depressive symptoms. Int J Methods Psychiatr Res. 2000;9:45-59.

62. Trivedi MH, Rush AJ, Ibrahim HM, Carmody TJ, Biggs MM, Suppes T, et al. The inventory of depressive Symptomatology, clinician rating (IDS-C) and self-report (IDS-SR), and the quick inventory of depressive Symptomatology, clinician rating (QIDS-C) and self-report (QIDS-SR) in public sector patients with mood disorders: a psychometric evaluation. Psychol Med. 2004;34:73-82.

63. Herdman M, Gudex C, Lloyd A, Janssen M, Kind P, Parkin D, et al. Development and preliminary testing of the new five-level version of EQ-5D (EQ-5D-5L). Qual Life Res. 2011;20:1727-36.

64. Bouwmans C, De JK, Timman R, Zijlstra-Vlasveld M, Feltz-Cornelis C, Tan SS, et al. Feasibility, reliability and validity of a questionnaire on healthcare consumption and productivity loss in patients with a psychiatric disorder (TiC-P). BMC Health Serv Res. 2013;13:217.

65. Derogatis LR, Melisaratos N. The brief symptom inventory: an introductory report. Psychol Med. 1983;13:595-605

66. Foa EB, Cashman L, Jaycox L, Perry K. The validation of a self-report measure of posttraumatic stress disorder: the posttraumatic diagnostic scale. Psychol Assess. 1997;9:445-51.

67. Horowitz LM, Alden LE, Wiggins JS, Pincus AL. Inventory of interpersonal problems. London: Psychological Corporation; 2000.

68. Schreurs PJG, Tellegen B, Vanderwillige G. Health, stress and coping-the development of the Utrechtse coping scale. Gedrag Tijdschrift Voor Psychologie. 1984;12:101-17.

69. Gratz KL, Roemer L. Multidimensional Assessment of Emotion Regulation and Dysregulation: Development, Factor Structure, and Initial Validation of the Difficulties in Emotion Regulation Scale. J Psychopathol Behav Assess. 2004;26(1):41-54.

70. Neumann A, van Lier PA, Gratz KL, Koot HM. Multidimensional assessment of emotion regulation difficulties in adolescents using the difficulties in emotion regulation scale. Assessment. 2010;17:138-49.

71. Ritschel LA, Tone EB, Schoemann AM, Lim NE. Psychometric properties of the difficulties in emotion regulation scale across demographic groups. Psychol Assess. 2015;27:944-54.

72. Watson D, Clark LA, Tellegen A. Development and validation of brief measures of positive and negative affect: the PANAS scales. J Pers Soc Psychol. 1988;54:1063-70.

73. Peeters FPML, Ponds RWHM, Vermeeren MTG. Affectiviteit en zeltbeoordeling van depressie en angst. Tijdschrift Voor Psychiatrie. 1996;38:240-50.

74. Engelen U, De Peuter S, Victoir A, Van Diest I, Van den Bergh O. Verdere validering van de Positive and Negative Affect Schedule (PANAS) en vergelijking van twee Nederlandstalige versies. Gedrag en Gezondheid. 2006;34:61-70.

75. van Rijsbergen GD, Bockting $C L$, Berking M, Koeter MW, Schene AH. Can a one-item mood scale do the trick? Predicting relapse over 5.5-years in recurrent depression. PLoS One. 2012;7:e46796.

76. van Rijsbergen GD, Burger H, Hollon SD, Elgersma HJ, Kok GD, Dekker J, et al. How do you feel? Detection of recurrent major depressive disorder using a single-item screening tool. Psychiatry Res. 2014;220:287-93.

77. Treynor W, Gonzalez R, Nolen-Hoeksema S. Rumination reconsidered: a psychometric analysis. Cogn Ther Res. 2003;27:247-59.

78. Raes F, Hermans D, Eelen P. De Nederlandstalige versie van de Ruminative Response Scale en de Rumination on Sadness Scale (The Dutch version of the Rumination Response Scale and the Rumination on Sadness Scale). Gedragstherapie. 2003;36:97-104.

79. Raes F, Hermans D. On the mediating role of subtypes of rumination in the relationship between childhood emotional abuse and depressed mood: brooding versus reflection. Depress Anxiety. 2008;25:1067-70.

80. Weissman AN, Beck AT: Development and validation of the dysfunctional attitude scale: a preliminary investigation. 1978. 
81. Beck AT, Brown G, Steer RA, Weissman AN. Factor analysis of the dysfunctional attitude scale in a clinical population. Psychol Assess J Consult Clin Psychol. 1991;3:478.

82. Nelson LD, Stern SL, Cicchetti DV. The dysfunctional attitude scale: how well can it measure depressive thinking? J Psychopathol Behav Assess. 1992;14: 217-23.

83. Oliver JM, Baumgart EP. The dysfunctional attitude scale: psychometric properties and relation to depression in an unselected adult population. Cogn Ther Res. 1985;9:161-7.

84. De Graaf LE, Roelofs J, Huibers MJ. Measuring dysfunctional attitudes in the general population: the dysfunctional attitude scale (form a) revised. Cogn Ther Res. 2009;33:345.

85. Pearlin LI, Schooler C. The structure of coping. J Health Soc Behav. 1978;2-21.

86. Pearlin LI, Menaghan EG, Lieberman MA, Mullan JT: The stress process. J Health Soc Behav 1981, 337-356.

87. Bernstein DP, Stein JA, Newcomb MD, Walker E, Pogge D, Ahluvalia T, et al. Development and validation of a brief screening version of the childhood trauma questionnaire. Child Abuse Negl. 2003;27:169-90.

88. Lecomte T, Corbiere M, Laisne F. Investigating self-esteem in individuals with schizophrenia: relevance of the self-esteem rating scale-short form. Psychiatry Res. 2006;143:99-108.

89. Costa PT, MacCrae RR: Revised NEO personality inventory (NEO PI-R) and NEO five-factor inventory (NEO-FFI): Professional manual. Psychological Assessment Resources, Incorporated; 1992.

90. Brugha TS, Cragg D. The list of threatening experiences: the reliability and validity of a brief life events questionnaire. Acta Psychiatr Scand. 1990;82: 77-81.

91. Horvath AO, Greenberg LS. Development and validation of the working alliance inventory. J Couns Psychol. 1989;36:223.

92. Larsen DL, Attkisson CC, Hargreaves WA, Nguyen TD. Assessment of client/ patient satisfaction: development of a general scale. Eval Program Plann. 1979;2:197-207.

93. Attkisson CC, Zwick R. The client satisfaction questionnaire. Psychometric properties and correlations with service utilization and psychotherapy outcome. Eval Program Plann. 1982;5:233-7.

94. Brooke J. SUS-A quick and dirty usability scale. Usability Eval Ind. 1996;189: 4-7.

95. Bangor A, Kortum PT, Miller JT. An empirical evaluation of the system usability scale. Intl J Human 「Çô Computer Interact. 2008:24:574-94.

96. Lipsey MW, Wilson DB. The efficacy of psychological, educational, and behavioral treatment: confirmation from meta-analysis. Am Psychol. 1993;48:1181.

97. Husereau D, Drummond M, Petrou S, Carswell C, Moher D, Greenberg D, et al. Consolidated health economic evaluation reporting standards (CHEERS) statement. BMJ. 2013;346:f1049.

98. Ramsey SD, Willke RJ, Glick H, Reed SD, Augustovski F, Jonsson B, et al. Cost-effectiveness analysis alongside clinical trials II-an ISPOR good research practices task force report. Value Health. 2015;18:161-72.

99. Zorginstituut Nederland 2016. Guideline for economic evaluations in healthcare. https://www.zorginstituutnederland.nl/over-ons/publicaties/ publicatie/2016/02/29/richtlijn-voor-het-uitvoeren-van-economischeevaluaties-in-de-gezondheidszorg. Accessed 21 Aug 2017.

100. Hoch JS, Rockx MA, Krahn AD. Using the net benefit regression framework to construct cost-effectiveness acceptability curves: an example using data from a trial of external loop recorders versus Holter monitoring for ambulatory monitoring of "community acquired" syncope. BMC Health Serv Res. 2006;6:68

101. Isaranuwatchai W, Markle-Reid M, Hoch JS. Adjusting for baseline covariates in Net benefit regression: How You adjust matters. Pharmacoeconomics. 2015;33(10):1083-90

102. Christensen H, Griffiths KM, Farrer L. Adherence in internet interventions for anxiety and depression: systematic review. J Med Internet Res. 2009;11(2): e13. doi:https://doi.org/10.2196/jmir.1194.

103. Kelders SM, Kok RN, Ossebaard HC, Van Gemert-Pijnen JE. Persuasive system design does matter: a systematic review of adherence to web-based interventions. J Med Internet Res. 2012;14(6):e152. doi:https://doi.org/10. 2196/jmir.2104.

\section{Submit your next manuscript to BioMed Central and we will help you at every step:}

- We accept pre-submission inquiries

- Our selector tool helps you to find the most relevant journal

- We provide round the clock customer support

- Convenient online submission

- Thorough peer review

- Inclusion in PubMed and all major indexing services

- Maximum visibility for your research

Submit your manuscript at www.biomedcentral.com/submit
Biomed Central 